\title{
Philosophiques
}

\section{Marc Henry Soulet, Le silence des intellectuels. Radioscopie de l'intellectuel québécois, Montréal, Éditions Saint-Martin, 1987.}

\section{Madeleine Ferland}

Volume 17, numéro 1, printemps 1990

URI : https://id.erudit.org/iderudit/027108ar

DOI : https://doi.org/10.7202/027108ar

Aller au sommaire du numéro

Éditeur(s)

Société de philosophie du Québec

ISSN

0316-2923 (imprimé)

1492-1391 (numérique)

Découvrir la revue

Citer cet article

Ferland, M. (1990). Marc Henry Soulet, Le silence des intellectuels. Radioscopie de l'intellectuel québécois, Montréal, Éditions Saint-Martin, 1987.

Philosophiques, 17(1), 143-149. https://doi.org/10.7202/027108ar d'utilisation que vous pouvez consulter en ligne.

https://apropos.erudit.org/fr/usagers/politique-dutilisation/ 
PHILOSOPHIQUES, Vol. XVII, Numéro 1, Printemps 1990

\section{ÉTUDE CRITIQUE}

MARC HENRY SOULET, Le silence des intellectuels. Radioscopie de l'intellectuel québécois, Montréal, Éditions Saint-Martin, 1987.

par Madeleine Ferland

L'intérêt premier de cet ouvrage tient à la pertinence de son sujet. Il est d'ailleurs symptômatique qu'il ait éré écrit par un Français ou - devrait-on dire - n'ait pas été écrit par un Québécois. Peut-être le milieu québécois n'était-il pas capable de se dire ce qu'il avait besoin d'entendre : les raisons de son silence et inévitablement une réflexion sur l'identité québécoise.

L'auteur cherchera à définir « le statut de la fonction intellectuelle dans une société d'après la modernité». En ce sens, l'analyse du silence des intellectuels se posera «en rapport à un état particulier des rapports sociaux » (p. 14) et voudra éviter des explications conjoncturelles ou morales.

\section{Médiation symbolique durant la modernité}

La première partie de l'ouvrage est théorique. Elle pose les postulats de la médiation symbolique comme fondement du rapport social et de la nécessité permanente du clerc comme médiateur. Elle se réfère à des thèses déjà connues, comme celle de Régis Debray dans Le scribe (1980) et à d'autres plus générales qui traitent de la transcendance et du sacré comme invariants sociaux. Citons Durkheim, Gauchet ou Lyotard dont les noms apparaissent dans la bibliographie. L'effort essentiel de cette partie portera sur la médiation symbolique propre à la modernité québécoise dans la mesure où l'auteur fait sien le postulat suivant lequel l'intellectuel québécois se manifeste seulement au moment où le Québec entre dans la modernité.

Pour définir l'intellectuel québécois, l'auteur cherchera ensuite à combiner la fonction permanente du clerc avec le cadre particulier de son origine sociohistorique. L'intellectuel sera caractérisé par «sa fonction génétique (le fait qu'il soit un produit social) et sa fonction générique (le fait qu'il exerce une œuuvre de transcendance) » (p. 33). A cette qualification, empruntée à G. Konrad et $Y$. Szelenyi, l'auteur en rajoure une troisième : l'autoréflexivité. C'est dans l'application de ces trois fonctions de l'intellectuel au contexte québécois que cette analyse prend toute sa pertinence. 
L'aufklärung québécoise s'est fondée sur la science, la langue et l'État. Dans les années cinquante, les élites traditionnelles, rurales, religieuses et protectionnistes, se sont heurtées à l'élite montante, composée de technocrates et de la nouvelle classe moyenne gagnée au discours de la modernité. Il faut se rappeler qu'à l'instar des sociétés dépendantes, l'absence d'une bourgeoisie autochtone a permis aux fractions supérieures de classes moyennes de s'imposer comme "nouvelle élite». Le nouveau pouvoir, fondé sur la rationalité et la compétence, a eu à constituer une infrastructure de production autour de l'État en plus de renouveler le discours nationaliste au nom d'un progressisme rationnellement organisé. Cette situation spécifique au Québec a donné aux intellectuels un rôle beaucoup plus important que le rôle idéologique qui leur aurait été dévolu autrement. D'abord hors de la cité, organisant leur discours dans les facultés de sciences sociales et critiquant fermement le nationalisme rétrograde de Duplessis, les universitaires québécois voient néanmoins l'État comme étant la seule solution pour parvenir à la modernisation escomptée. Donc quand les libéraux accèdent au pouvoir, la voie est toute tracée pour ces intellectuels qui, avec l'accroissement de la légitimité des sciences sociales, deviennent les compétences indispensables à l'édification du Québec moderne. S'ouvrent par le fait même des secteurs d'emploi nouveaux : 1- dans l'appareil scolaire, quand la conception de l'éducation s'identifie aux visées de l'État et offre donc la possibilité d'une promotion sociale; 2 - dans la fonction publique qui se développe en négligeant le secteur privé parce qu'on lui reproche d'être demeuré capitaliste et sourd au nouveau discours nationaliste : 3 -dans la recherche universitaire, où les sciences sociales connaissent un développement inouï, dans la mesure où alliant science et polirique, elles légitiment le fondement technocratique de la rationalité de l'État qui en retour légitime les sciences sociales comme discours scientifique privilégié.

Le «marché» des intellectuels a tout de même connu des moments distinctifs: (l'auteur emprunte à S. Proulx cette catégorisation) en 1950, l'idéologie est au rattrapage, ce qui explique le rôle réformiste de l'élite intellectuelle d'alors, qui a dú faire de la politique et de l'administration pour entièrement rebâtir les institutions; les années 1970 sont celles de la contestation, qui n'aura pu se faire qu'à l'université, sur un discours de gauche très radical et par une nouvelle génération réduite au discours de la subversion pour se démarquer de celui de la génération précédente, déjà en fonction dans les instances gouvernementales; finalement, les années 1980 débouchent sur les intellectuels de l'incertitude, la génération précédente ayant monopoliséà la fois le rôle de la raison et celui de sa critique, tout en ayant soutenu et perdu l'idéologie nationaliste et socialiste à travers le Parti Québécois et les groupes marxistes-léninistes. C'est la génération des pigistes, qui n'ont pas de discours spécifique, pas de légitimité, pas de place ni à l'université, ni dans la fonction publique. Cette génération de l'incertitude constitue le problème de l'ouvrage.

De façon générale, il faut retenir que l'avènement de l'intellectuel québécois était nécessairement lié à la question nationale («bâtisseur du 
Québec ») et que ses traits spécifiques au cours des trente dernières années populisme et fantasme de l'unité - dépendront toujours de ce premier ancrage. Après le référendum, l'intellectuel québécois se retrouvera incapable de créer un discours, à cause du rôle de représentant du peuple qu'il se sentait le devoir d'assumer. D'un côté, les marxistes-léninistes développent une idéologie anti-intellectualiste, d'un autre, les libéraux fédéralistes accusent les intellectuels québécois de ne pas avoir respecté les désirs du peuple. S'ajoute la culpabilité de ses origines : intellectuel sans tradition intellectuelle, «parvenu» sans avoir pu assurer la légitimité de sa fonction, il intériorise, tout en la combattant, la coupure avec le peuple. D'où une humilité et un mépris de soi par rapport à son entourage et aussi vis-à-vis de la tradition intellectuelle étrangère.

La deuxième partie de l'ouvrage porte sur les causes du silence des intellectuels québécois, qui ne peuvent être comprises en dehors de l'analyse du changement des rapports sociaux qui ont signé l'échec du projet de la modernité du Québec. L'auteur distingue trois aspects à son analyse : 1- la «déflation idéologique » 2- «la surfonctionnalisation de la société » 3- «la crise de transmission des acquis» respectivement responsables de trois ruptures dans la possibilité d'être de l'intellectuel québécois : 1- la disjonction entre « l'objet et la chose » 2 - la disjonction entre "le social brut et le social construit » 3- la disjonction entre « le confort et la précarité ». Cette partie débouchera naturellement sur une dernière partie portant sur la « radioscopie proprement dite de l'intellectuel québécois » dans la société postmoderne, l'une étant explicative de l'autre. C'est dans le lien causal entre ces deux dernières parties (entre les rapports sociaux et la fonction génétique des intellectuels québécois) que réside toute la pertinence et l'originalité de cet ouvrage.

\section{Changements dans les rapports sociaux}

Le premier point abordé par l'auteur dans cette deuxième partie est le passage de la question nationale au retrait du politique. Comme la politique passait par l'idée d'indépendance, la défection vis-à-vis de l'une entraîna irrémédiablement celle vis-à-vis de l'autre, le référendum étant le point charnière autour duquel se préciseront des problèmes insolubles au projet émancipateur du Québec. Les intellectuels de gauche avaient reporté leur projet socialiste et leurs préoccupations sociales au moment de l'indépendance, contribuant ainsi à créer l'illusion d'un consensus politique qu'ils n'ont pu reconstituer par la suite, après l'échec du $P Q$; le mouvement syndical aurait fait la même erreur en misant toute sa stratégie sur le rôle émancipateur du secteur public et le projet transclassiste du $P Q$, sans penser sa relation avec le secteur privé et sa rupture de plus en plus marquée avec les groupes populaires. La politique s'est coupée du « vécu » pour devenir une représentation médiatique formelle. $A$ partir du référendum, l'écart entre «le mot et la chose » apparaît au grand jour, retirant du coup la légitimité du discours aux 
intellectuels. D'après Soulet, toute la stratégie du PQ serait responsable du retrait obligé du politique. Selon lui, l'indépendance aurait dû demeurer symbolique, car en passant au politique, elle déresponsabilise le peuple, (en confiant l'idéologie à l'État), dont l'histoire et la pratique a toujours consisté à jouer sur les deux tableaux de son «ubiquité originelle » : souveraine dans le cceur, fédéraliste quand il le faut. Le projet de société aurait donc reposé sur une illusion juridique et linguistique (la loi 101 par exemple), qui n'aura réussi qu'à dénaturer et appauvrir le projet collectif. Le peuple a manifesté qu'il ne voulait pas de l'indépendance et de façon générale d'une société reposant sur le politique.

Le second aspect du silence des intellectuels est la surfonctionnalisation de la société, inévitable et condamnable à la fois selon Soulet, parce que le Québec moderne ne pouvait advenir sans tout investir dans l'État, et que cet investissement ne pouvait mener qu'à un autoritarisme administratif fondé sur la recherche rationnelle et objective, à l'encontre d'une politisation des problèmes sociaux dont aurait pu être saisie la société civile. D'après cette analyse, Soulet en arrive à interpréter le passage de la période hautement politique des années 1960 à celle du repli pragmatique des années 1980 comme une même continuité dans la rationalité technocratique. L'illusion résiderait dans le fait que, durant les années soixante, les intellectuels étaient les auteurs du discours de légitimation de l'État gestionnaire, alors que dans les années 80 , avec la gestion de la "décroissance", ils en deviennent les premières cibles, le rôle de médiation symbolique qui avait fait leur légitimité s'étant progressivement déplacé vers les fonctionnaires experts, formés dans les services internes de l'État, et vers une nouvelle caste médiatique: les journalistes politiques professionnels. Selon l'auteur, les intellectuels portent néanmoins la responsabilité de leur choix promotionnel de la rationalité scientifique au détriment de la culture antérieure et de la prise en charge du quotidien par le citoyen. En renonçant à réconcilier le peuple avec son sens historique, ils auraient créé une disjonction entre le social brut et le social construit.

Le troisième aspect du silence des intellectuels est la crise de transmission des acquis. Celle-ci serait due à l'incapacité de cette classe à se reproduire et au refus agressif de la nouvelle génération d'accepter des valeurs qu'elle considère comme des privilèges immérités. En fait, autant les «vieux» que les « jeunes» substituent au projet collectif un repli sur soi narcissique. $A$ ces explications globales, l'auteur apporte tout de même un détail intéressant quant à la situation québécoise : suivant son hypothèse, la crise de transmission des acquis ne serait pas due à l'embourgeoisement de la classe intellectuelle, comme on le prétend généralement, mais bien à l'arrêt de l'embourgeoisement dans la fonction publique, le résultat étant un hiatus entre l'identification aux visées de l'État et les aspirations individuelles de promotion sociale. C'est ainsi que se constitue la disjonction entre le confort et la précarité, que ne peut dénoncer l'intellectuel « fortement rétribué » (p. 140) sans se salir en même temps au passage, car il risquerait d'y perdre matériellement mais 
surtout symboliquement. L'intellectuel ne peut plus tenir de discours normatif sur le monde. Dorénavant il n'a plus d'aurres interlocuteurs que ses collègues universitaires et sa fonction se limite à former des professionnels.

\section{Fonction génétique des intellectuels québécois dans une société postsociale}

L'analyse du changement dans les rapports sociaux permet maintenant à Soulet de questionner le sort de l'intellectuel dans la société postmoderne québécoise. Il se demande initialement «comment se gère la médiation symbolique au sein de cette société devenue autoréférentielle», dans la mesure où la représentation d'une société à elle-même se limite à une «représentation théâtralisée du partage d'une même condition» (p. 153). Pour les raisons que l'on veut, allant d'une conception ouvriériste ou romantique de l'intellectuel qui a trahi les siens, à la position de Lyotard concernant la fin des «Grands Récits » où l'avenir est à construire sur la communication, en l'absence de tout principe référentiel (Soulet en fait une simple énumération), une telle société n'a plus besoin de clercs, car le rôle de médiation qui leur était dévolu dans les sociétés référentielles est ici remplacé par la communication intersubjective. Or cette personnalisation des rapports sociaux porte la fin de l'espace public, car elle dissout le politique dans le social et le social dans l'immédiateté de la communication. La seule médiation possible s'organise autour de l'insécurité constitutive à la perte d'un référent, et sa théâtralisation sert de lien social, même si toutes ses représentations sont sans contenu politique.

Que peut faire l'intellectuel s'il ne possède plus de lieu de discours autorisé ? Soulet présente les différentes avenues qu'il a puisées dans son étude empirique, mais il omer de les mettre à discussion, ce qui aboutit à une certaine confusion entre les témoignages recueillis et les thèses de l'auteur parmi lesquelles certaines propositions normatives auraient mérité d'être davantage étudiées. On ne sait pas, par exemple, comment interpréter la responsabilité des intellectuels du $\mathrm{PQ}$ d'avoir brisé le rêve québécois «au point de faire du nationalisme simultanément un outil de libération et un instrument de domination » ni dans quelle mesure on peut accuser l'idéologie nationaliste, constituée sur l'inconscient collectif du colonisé, d'avoir empêché toute forme de pensée ou de vie intellectuelle. Distinguons tout de même quatre positions essentielles : 1-certains intellectuels réitèrent le discours des Lumières et rêvent de retourner dans l'opposition pour légitimer leur parole ; 2 - d'autres s'interrogent sur la possibilité d'une médiation symbolique " en creux " du discours officiel et qui pourrait se constituer d'un autre lieu que de la sphère politique désormais considérée comme responsable d'un consensus aliénant établi au nom de la modernité ; il s'agit surtout de parler du social, du quotidien, pour lui donner un ancrage politique mais en refusant tout projet global pour la société ; 3- la plupart se convertissent en experts professionnels apolitiques, (de là s'expliquerait un désinvestissement visà-vis des sciences sociales normatives comme la sociologie ou l'anthropologie 
et un regain d'intérêt pour les disciplines pratiques comme l'économie et l'administration) ; 4- certains - et c'est la voie qui semble privilégiée par Soulet - assument de se faire les simples narrateurs des forces sociales en cours.

Dans toute leur diversité, ces positions se rejoignent en ce qu'aucune n'est émettrice de sens, ce qui oblige tous les intellectuels à se résoudre à un retour sur soi qui pourra prendre deux formes: la «culture cultivée» et la «culture de soi». Pour remplacer la raison fondatrice, les intellectuels ont besoin de se rattacher une "généalogie académique - c'est la "culture cultivée »- qui les légitime comme savants, et même, ajoute Soulet, qui justifie leur démission. D'où un retour à la tradition classique, condition négligée des intellectuels québécois. D'un autre côté, la «culture de soi » sert à désamorcer leur situation de parvenus, devenue évidente à partir du moment où ils ne sont plus fondés de parole. Ils se revendiquent auteurs, et se déchargent de leur implication pédagogique. Ils développent des intérêts artistiques qui leur permettent de déplacer leur champ discursif du politique au culturel. Ils rejettent la conception engagée de l'intellectuel qui les plaçait en jonction constante avec le peuple pour se définir à l'intérieur d'une élite savante.

Au terme de son analyse, Soulet dégage deux voies possibles pour recomposer le rôle de l'intellectuel québécois : celle de l'honnête homme se réclamant d'une position éthique et qui refusera le confinement dans la pédagogie ou la spécialité ; celle du communicateur hors de l'institution qui assurera une « dimension stratégique de travailleur de surface » et que pourra sûrement mieux assumer la génération intellectuelle non instituée.

En conclusion, Soulet prône la nécessité pour l'intellectuel de se penser autrement pour permettre à la communauté de se fonder, non pas un sens collectif mais un lien entre la culture de soi et la culture civique. L'intellectuel trouverait là un rôle essentiel de publicité des normes de l'agir humain en conciliant des normes collectives qui sauront former une volonté générale et la multitude des valeurs et intérêts. La fonction intellectuelle se définirait donc toujours comme «élucidation des enjeux éthiques et politiques de l'existence sociale» (p. 206) mais où le fondement du sens collectif serait à construire dans la délibération publique. Or, la société intimiste empêche actuellement toute possibilité de débat public en annulant toute manifestation publique des différences et en inscrivant l'individu dans l'ordre strictement instrumental du social. Soulet critique l'intimisme comme une illusion démocratique qui, en favorisant l'individu au détriment du citoyen, prive celui-ci de son espace de parole autorisée - l'espace public - en le vidant de tout contenu. Il se distingue de la position habermassienne en ce qu'il refuse la notion de vérité préétablie comme fondement de l'être-ensemble, et laisse ouverte la question de savoir où et comment pourra se faire la délibération publique.

La nouvelle fonction de l'intellectuel défendue par Soulet prolonge l'image idéalisée de l'intellectuel de la modernité. Cette position relève d'un 
choix théorique de l'auteur qui n'a pas retenu comme caractéristique du rôle de l'intellectuel, son nécessaire rapport avec le pouvoir. Cette omission lui a permis de jouer sans cesse sur une ambiguité entre la fonction générique de l'intellectuel et sa fonction génétique dans la modernité. Ce qui l'empêche d'admettre qu'en conséquence de sa propre analyse, les intellectuels du Québec postmoderne pourraient maintenant former la nouvelle classe des professionnels de la communication : psy de tour acabit, journalistes, analystes et chroniqueurs de la presse écrite et électronique, hauts fonctionnaires et organisateurs politiques, chanteurs populaires engagés etc. On comprend néanmoins le désir de l'auteur de faire porter le questionnement sur des possibilités d'être de l'intellectuel qui puisse penser l'image de l'unité de la communauté en des termes plus progressistes, sans nostalgie pour la modernité et en correspondance intime avec le peuple. Or le concept de peuple n'est-il pas à redéfinir avec l'ouverture du Québec à son impensé Américain et la découverte tardive des nouvelles communautés culturelles québécoises? Tantôt internationaliste sans identité, tantôt identifié par sa négation du regard canadien, le « nous " québécois ne pourra plus se constituer dans l'imaginaire social défini par le référendum. Mais la « faute » en revientelle uniquement aux intellectuels d'alors comme le laisse entendre Soulet, qui ne revient d'ailleurs jamais sur ce point névralgique du refoulé québécois? $\mathrm{Si}$ les intellectuels au pouvoir ont fait de la "politique théorique " plutôt que "pratique» en basant leurs efforts sur des transformations juridiques et linguistiques, il est néanmoins difficile de concevoir ce qu'aurait pu être une responsabilité d'intellectuel qui refuse le pouvoir d'État. On peut toutefois reconnaître la justesse de sa critique du pouvoir technocratique de la modernité tout en tentant de la situer autrement dans l'histoire québécoise. Suggérons la possibilité d'inscrire cette étape comme l'avènement d'un pouvoir technocratique qui s'est implanté au nom des idées modernes de justice et de raison et non comme la seule véritable naissance du Québec à travers l'idéal de la modernité et qu'on aurait trahi par la suite. Finalement, on se demande si la critique du PQ n'est pas finalement celle de la modernité, québécoise ou internationale, et si celle de la postmodernité doit être imputée à l'irresponsabilité des intellectuels qui ont noyé l'imaginaire social en tentant de le réaliser politiquement. Cette confusion aurait peut-être pu être évitée si l'auteur avait intégré des éléments de comparaison entre la modernité québécoise et l'histoire européenne et américaine. L'ouvrage aura tout de même permis de profiter d'une analyse empirique du contexte québécois qui garde toute sa pertinence et suggéré dans son positionnement clair vis-à-vis de la nécessité d'une pensée engagée, une lutte, essentielle dans les circonstances, contre l'anti-intellectualisme.

Département de philosophie, Collège Montmorency 